\title{
PRESENTACIÓN DE LA SECCIÓN TENDENCIAS NUEVOS TIEMPOS, NUEVOS RETOS
}

doi: https://doi.org/10.26439/contratexto2021.n036.5574

Empezamos el año 2021 con la grata noticia de la indexación de Contratexto en dos de las más importantes bases de datos científicas: Scopus y SciELO Perú1. De esta experiencia obtenemos lecciones y desafíos para asegurar un proceso editorial riguroso. Una de esas lecciones es la necesidad de consolidar el comité científico de pares revisores. De su aporte depende la calidad de cada número de la revista y su selección no solo debe corresponder a su experiencia, dominio temático y grados académicos, sino también a fomentar una comunicación asertiva que contribuya a enriquecer las propuestas y fortalecer la colaboración de la comunidad científica.

Otro desafío que se nos presenta es reflexionar sobre el rasgo distintivo de Contratexto en la producción científica de la investigación en comunicación. En ese sentido, deseamos priorizar la producción de investigaciones empíricas que movilicen las fronteras de nuestra disciplina o ensayos que ofrezcan perspectivas nuevas y fructíferas polémicas.

Aprovechamos esta oportunidad para rendir un merecido homenaje a tres investigadores de la Comunicación que nos han dejado estos meses. Colegas cuyos aportes marcaron el devenir de nuestra profesión: Rafael Roncagliolo, Jesús Martín Barbero y Enrique Bustamante. Los dos primeros, autores de Contratexto, y el tercero, quien desde la revista Telos influyó notablemente en el quehacer intelectual iberoamericano. La impronta de los tres resulta decisiva en el desarrollo del campo disciplinar y, por ello, nuestra gratitud.

La sección Tendencias ha cobrado un inusitado interés en esta nueva etapa de Contratexto. Abre la sección el trabajo de Juan Pablo Arrobo-Agila, Abel Suing e Ignacio Aguaded titulado "Narrativas transmedia en la comunicación de catástrofes: el terremoto de Ecuador del 2016", donde se analizan las publicaciones sobre este hecho en dos

1 Red regional liderada en el Perú por el Consejo Nacional de Ciencia, Tecnología e Innovación Tecnológica (CONCYTEC), la Universidad Nacional Mayor de San Marcos y la representación nacional de la Organización Panamericana de la Salud. 
diarios en Facebook señalando los retos en materia educativa y preventiva. En la misma línea comparativa, tenemos el estudio de Sebastián-Alberto Longhi-Heredia, Laddy Liset Quezada-Tello y Giancarlo Capello, quienes comparan las estrategias digitales de difusión de los museos en Ecuador, España y Perú en el contexto de la pandemia del COVID-19. A la luz de los lineamientos planteados por la UNESCO, encuentran variaciones a nivel supranacional y patrones temáticos de carácter comunicacional y educativo.

Desde Brasil, Amanda Cantú Rodrigues Soares y Jane Márcia Mazzarino abordan la contribución de las redes sociales al desarrollo de la Cuarta Ola Feminista en ese país. A partir de una revisión bibliográfica y el análisis del activismo virtual de movimientos feministas, se centran en el caso "Não Me Kahlo" para demostrar la importancia de las redes para la comunicación, organización y divulgación de sus contenidos, así como los retos que representa. Cierra la sección el trabajo de José Carlos Cabrejo Cobián (Perú), quien nos ofrece un ensayo que toma el concepto de quiasmo de Merleau-Ponty y aportes de la semiótica generativa y tensiva para comprender las dimensiones surrealistas enfocadas en la entidad cuerpo/mundo expresado tanto en la obra cinematográfica de Buñuel como en la poesía de César Moro.

El próximo número de la revista tendrá un sentido especial. La Facultad de Comunicación de la Universidad de Lima, institución que concibió y publica esta revista desde hace más de tres décadas, cumple cincuenta años al servicio de la formación de comunicadores. Es por ello que queremos reflexionar sobre esta labor en Iberoamérica a la que dedicamos un dosier que estará a cargo de la doctora Teresa Quiroz. La convocatoria prevé un espacio para esbozar ideas y reflexiones críticas que nos permitan comprender los cambios, tensiones y desafíos para el desarrollo profesional de nuestra disciplina desde las aulas.

Queremos agradecer a Franahid D'silva, de la Universidad Nacional Abierta (Venezuela), y Mariana Gabriela Torres, de la Universidad Nacional de la Patagonia Austral (Argentina), editoras invitadas del dosier "Las nuevas formas de comunicación educativa: aula extendida", que incluye este número.

Esperamos que disfruten de su lectura.

Julio César Mateus

Director

Lilian Kanashiro

Editora general 